\title{
PLANTAS EMPLEADAS EN MEDICINA TRADICIONAL EN TIERRA CALIENTE, GUERRERO, MÉXICO PARA EL TRATAMIENTO DE ENFERMEDADES INFECCIOSAS
}

\section{ETHNOMEDICAL PLANTAS TO TREAT INFECTION DISEASES USED IN TIERRA CALIENTE, GUERRERO, MEXICO}

\author{
ROJAS, H, NIDIA M ${ }^{1 *}$ Ph.D., AVELLANEDA, S. SENOVIO² Ph.D., \\ CUÉLLAR CUELLAR ARMANDO ${ }^{3}$ Ph.D.
}

${ }^{1}$ Investigadora Titular, Departamento de Microbiología, Facultad de Biología, Universidad de la Habana, Calle 25 № 455, Vedado, La Habana, Cuba. Facultad de Zootecnia y Veterinaria, Universidad de Guerrero, Guerrero, México (Q.E.P.D). ${ }^{3}$ Departamento de Farmacia, Instituto de Farmacia y Alimentos, Universidad de la Habana.

* Correspondencia: nidia.rojas@infomed.sld.cu

Recibido: 29-11-2009; Aceptado: 12-03-2010

\section{Resumen}

La región Tierra Caliente, del estado de Guerrero, México, posee una variada flora, parte de la cual se utiliza por sus pobladores para el tratamiento de sus enfermedades. Sin embargo, existe poca información sobre las plantas medicinales utilizadas en esta zona y sobre cuáles se emplean para el tratamiento de las enfermedades infecciosas. Con el objetivo de obtener información sobre las plantas utilizadas en la medicina tradicional de esta región para curar enfermedades de origen microbiano, se realizó una encuesta etnomedicinal de tipo mixto con una pregunta abierta y ocho cerradas a personas conocedoras de la flora local. Se seleccionaron 30 planillas referentes a plantas empleadas para el tratamiento de enfermedades de posible origen microbiano. La encuesta permitió identificar nueve plantas usadas con esta finalidad, las que fueron identificadas taxonómicamente según las características de material vegetal herborizado: Karwinskia humboldtiana Roem et Sch. Zucc. (Guayabillo), Waltheria indica L. (Güinar), Zizyphus amole (Seesé \& Moc) M.C. Jhonst (Corongoro), Cyrtocarpa procera Kunth in H. B. (Chucumpún), Lippia alba N.E. Brown ex Britton \& Wilson (Tarape), Acacia farnesiana L. Willd (Huizache), Diphysa minutifolia Rose (Shure), Euphorbia albomarginata Torr \& Gray (Golondrina) y Curatella americana L. (Rasca Viejo). Se determinó la parte de la planta empleada, el modo de preparación y la dosificación, tiempo de empleo, así como sus aplicaciones. Las plantas C. procera, $Z$. amole y $D$. minutifolia que no se han reportado anteriormente como medicinales.

Palabras Clave: Encuesta etnomedicinal, plantas medicinales, propiedades antimicrobianas. 


\section{Abstract}

In Tierra Caliente region, Guerrero state, Mexico, it is possible to find a great variety of species of plants used in traditional medicine, but only few of them are scientifically studied in particular for antimicrobial activity. In order to obtain information about the plants used in this region are the plants used in traditional medicine in this region to treat infection diseases it was done an ethnomedical survey mixed with one open and eight closed questions to traditional practitioners. From this survey 30 microbial were selected which included plants used to treat infection diseases. Nine species were recognized and botanically characterized: Karwinskia humboldtiana Roem et Sch. Zucc. (Guayabillo), Waltheria indica L. (Güinar), Zizyphus amole (Seesé \& Moc) M.C. Jhonst (Corongoro), Cyrtocarpa procera Kunth in H. B. (Chucumpún), Lippia alba N.E. Brown ex Britton \& Wilson (Tarape), Acacia farnesiana L. Willd (Huizache), Diphysa minutifolia Rose (Shure), Euphorbia albomarginata Torr \& Gray (Golondrina) y Curatella americana L. (Rasca Viejo). It was recorded the part of the plant, the way of preparation, dossification and the extention of the treatment and how was applied in the traditional use. $C$. procera, $Z$. amole and $D$. minutifolia were recorded for the first time as medicinal plants.

Key words: ethnomedical survey, medicinal plants, antimicrobial properties

\section{Introducción}

A través de la historia, las plantas han proporcionado a los seres humanos una fuente renovable de alimentos y medicamentos. La medicina tradicional y su práctica han permitido conocer el uso de plantas curativas para el tratamiento de diversas patologías desde los primeros albores de la humanidad (MORÓN, 2006), pero desde hace poco más de un siglo, el desarrollo científico-técnico posibilitó validar el empleo de muchas de estas especies vegetales para los sistemas primarios de salud (MECKES et al., 2004; CÁCERES, 2005).

La región de Tierra Caliente, se encuentra ubicada geográficamente al Noreste del Estado de Guerrero, México, colinda al norte con el Estado de Michoacán, al Sureste con Teycpan y Atoyac de Alvarez y al Oeste con Coahuayutla de Guerrero (INEGI, 2000). Su población, mayoritariamente rural emplea las plantas de forma sistemática para el tratamiento de sus enfermedades y dolencias, a veces por razones económicas y en otras por 
tradición familiar. Sin embargo, no se encontraron publicaciones anteriores en las que se haga referencia a las plantas medicinales empleadas en la región de Tierra Caliente o se haya realizado un levantamiento de su uso terapéutico, ni tampoco sobre las plantas que se utilizan como remedios para enfermedades de origen microbiano, lo que justifica la realización del presente trabajo.

Por todo lo anterior, el objetivo propuesto fue determinar mediante una encuesta etnomedicinal de las plantas empleadas por la población de esa región en la medicina natural para el tratamiento de enfermedades de origen microbiano y las especificaciones de su uso.

\section{Materiales y métodos}

Descripción de la encuesta: Se elaboró y aplicó una encuesta etnomedicinal de tipo mixto (SANDOVAL, 1998), dirigida o programada, con una pregunta abierta para declarar el nombre común por el cual se conoce a la planta en esa región y ocho preguntas cerradas de respuesta múltiple con diversas opciones probables impresas en planillas individuales (una por planta) que se entregaban a cada persona entrevistada.

Entre las preguntas incluidas en la encuesta se encontraban:

1- Parte de la planta usada

2- Forma de preparación

3- Dosis recomendada

4- Días de uso

5- Enfermedades para las que se recomienda

6- ¿Cómo conoció acerca de las propiedades de esa planta?

7- ¿Cómo la adquirió?

8- ¿Qué tipo de planta es?

Se recogieron 150 planillas con información sobre las plantas más frecuentemente empleadas en la medicina tradicional de Tierra Caliente. 
Para llenar las planillas en cada municipio se seleccionaron a informantes claves, como el subgrupo de integrantes del territorio que según criterios de personal confiable constituían las mejores fuentes de información, tomando como criterio que las personas escogidas conocieran de plantas medicinales, que estuvieran en disposición de brindar información y que fueran representativas por sus conocimientos relacionados con la medicina tradicional en su comunidad (PINEDA et al., 2000).

Estos informantes eran campesinos, yerberos, curanderos y personas conocedoras de las plantas de la región que pertenecían a los nueve municipios de Tierra Caliente: Ajuchitlán del Progreso, Arcelia, Cutzamala de Pinzón, Coyuca de Catalán, Pungarabato, San Miguel Totoloapan, Tlalchapa, Tlapehuala, y Zirándaro de los Chávez.

Cuando las personas encuestadas no sabían leer, se les leyó la planilla con el cuestionario de la encuesta para garantizar su participación y se llenó en su presencia con las respuestas dadas a cada pregunta.

Recolección del material vegetal: Se colectaron muestras frescas de cada planta mencionada, se herborizaron y depositaron en el Instituto de Biología y en el Laboratorio de Plantas Vasculares de la Facultad de Ciencias de la Universidad Nacional Autónoma de México (UNAM) para su identificación taxonómica, según la metodología existente en esas instituciones para esos fines. Las plantas se recolectaron en los meses de julio y agosto del año 2001 en el horario comprendido entre las nueve y diez de la mañana. La colecta se realizó de acuerdo con los nombres comunes por los cuales se reconocen las plantas en la región y en los mismos municipios donde se recomendaron como medicinales.

\section{Resultados}

Según las planillas de la encuesta, existen nueve plantas que se emplean en Tierra Caliente para el tratamiento de diferentes enfermedades, entre las cuales se encuentran enfermedades de etiología microbiana. Las plantas señaladas se conocen en esta región por los nombres comunes de: Guayabillo, 
Güinar, Corongoro, Chucumpún, Tarape, Huizache, Shure, Golondrina y Rasca Viejo.

Una vez identificadas taxonómicamente, estas plantas correspondieron a las especies y familias botánicas que se presentan en la Tabla 1.

Tabla 1. Identificación taxonómica de las plantas empleadas en medicina tradicional en Tierra Caliente para el tratamiento de enfermedades infecciosas según la encuesta realizada

\begin{tabular}{|l|l|l|}
\hline Nombre común & \multicolumn{1}{|c|}{ Nombre científico } & \multicolumn{1}{|c|}{ Familia } \\
\hline Guayabillo & Karwinskia humboldtiana Roem et Sch. Zucc & Rhamnaceae \\
\hline Güinar & Waltheria indica L. & Esterculiaceae \\
\hline Huizache & Acacia farnesiana L. Willd. & Mimosaceae \\
\hline Corongoro & Zizyphus amole (Seesé \& Moc) M.C. Jhonst & Rhamnaceae \\
\hline Chucumpún & Cyrtocarpa procera Kunth in H. B. & Anacardiaceae \\
\hline Tarape & Lippia alba N.E. Brown ex Britton \& Wilson & Verbenaceae \\
\hline Shure & Diphysa minutifolia Rose & Fabaceae \\
\hline Golondrina & Euphorbia albomarginata Torr. \& Gray & Euphorbiaceae \\
\hline Rasca Viejo & Curatella americana L. & Dilleniaceae \\
\hline
\end{tabular}

Como se observa en la tabla 2, la planta más empleada por los entrevistados fue el Corongoro, citada en el $20 \%$ de las planillas evaluadas en este trabajo, La corteza de esta planta reducida a polvo o macerada se aplica en uso externo para el tratamiento de heridas y para afecciones de la piel fundamentalmente, aunque la planta conocida como Tarape también se recomienda frecuentemente $(13,3 \%)$ por los pobladores de esta región.

Tabla 2. Datos recogidos de las plantas seleccionadas a partir de la encuesta

\begin{tabular}{|l|c|l|c|c|l|l|}
\hline $\begin{array}{c}\text { Nombre } \\
\text { común }\end{array}$ & $\begin{array}{c}\text { \% de planillas } \\
\text { que las } \\
\text { recomiendan }\end{array}$ & $\begin{array}{c}\text { Forma de } \\
\text { Preparación }\end{array}$ & Administración & $\begin{array}{c}\text { Días de } \\
\text { uso }\end{array}$ & $\begin{array}{c}\text { Parte de la } \\
\text { planta } \\
\text { usada }\end{array}$ & Recomendada para \\
\hline Guayabillo & 10 & Infusión & Oral & $1-3$ & Hojas y Tallo & Diarrea \\
\hline Güinar & 10 & Infusión & Oral & $3-7$ & Raíz & Diarrea \\
\hline Corongoro & 20 & $\begin{array}{l}\text { Macerado, } \\
\text { Polvo }\end{array}$ & Externo & $3-7$ & Corteza & $\begin{array}{l}\text { Heridas, Infecciones de } \\
\text { la piel }\end{array}$ \\
\hline Chucumpún & 10 & Infusión & Oral & Más de 7 & Corteza & Infecciones renales \\
\hline
\end{tabular}




\begin{tabular}{|l|c|l|c|c|l|l|}
\hline Tarape & 13,3 & Infusión & Oral & $1-3$ & $\begin{array}{l}\text { Hojas y y } \\
\text { Tallo }\end{array}$ & Diarrea \\
\hline Huizache & 10 & Infusión & Oral & $3-7$ & Raíz & Diarrea \\
\hline Shure & 10 & $\begin{array}{l}\text { Infusión, } \\
\text { Macerado }\end{array}$ & Oral & $1-3$ & Corteza & Diarrea \\
\hline Golondrina & 10 & Infusión & Oral & Más de 7 & Hojas y Tallo & Infecciones renales \\
\hline Rasca Viejo & 6,6 & $\begin{array}{l}\text { Macerado, } \\
\text { Infusión }\end{array}$ & Oral & 7 & Corteza & $\begin{array}{l}\text { Infecciones renales, } \\
\text { Cólicos }\end{array}$ \\
\hline
\end{tabular}

De acuerdo con estos resultados, el 88,8 \% de estas plantas se usan de forma oral, por lo que es lógico que se preparen como cocimiento o infusión. Para el uso externo se utilizan en forma de macerados $(33,3 \%)$ o directamente reducidas a polvo (11,1\%). En general, las partes de las plantas empleadas son las hojas y tallos (33\%) y raíces (22\%), así como la corteza del tronco (44\%). No se hace referencia acerca del empleo de flores o frutos con fines curativos.

En la Tabla 3 se observa que las plantas señaladas en la encuesta por su uso en las enfermedades de posible origen microbiano pueden ser árboles, arbustos o hierbas, que se conocen por tradición familiar o regional y que en la mayoría de los casos, la recogen del campo, aunque a veces algunas existen en los patios y jardines de las viviendas, pero en ningún caso fueron compradas a yerberos.

Tabla 3. Datos acerca del tipo de planta, su adquisición y forma en que la conoció el encuestado

\begin{tabular}{|l|l|}
\hline \multicolumn{1}{|c|}{ Datos } & \multicolumn{1}{c|}{ Resultados (\%) } \\
\hline Tipo de planta & $\begin{array}{l}\text { Árbol }(36,6) \\
\text { Arbusto }(30,0) \\
\text { Hierba }(33,3)\end{array}$ \\
\hline Conocimiento de la planta & $\begin{array}{l}\text { Tradición familiar }(50) \\
\text { Tradición regional }(50)\end{array}$ \\
\hline Obtención de la planta & $\begin{array}{l}\text { La compró }(0) \\
\text { Se la regalaron }(6,6) \\
\text { La recogió del campo }(93,3)\end{array}$ \\
\hline
\end{tabular}

La sinonimia de nombres comunes relacionados con las plantas medicinales halladas en este estudio se presenta en la tabla 4. 
Tabla 4. Clasificación taxonómica de otras especies vegetales conocidas con los mismos nombres comunes en México, según Martínez (1994)

\begin{tabular}{|c|c|c|}
\hline Nombre común & \multicolumn{2}{|c|}{ Nombre científico } \\
\hline Guayabillo & $\begin{array}{l}\text { Casearia arguta H.B.K } \\
\text { Calyptranthes Berg } \\
\text { Caseria silvestris Swartz } \\
\text { Dodonaea viscose Jacq. } \\
\text { Eugenia fragans Swartz } \\
\text { Willd.. }\end{array}$ & $\begin{array}{l}\text { Eugenia guatemalensis Don } \\
\text { Eugenia sinaloae Stand } \\
\text { Gimora nudiflora Hems Koehne } \\
\text { Lopezia racemosa Jacq. } \\
\text { Xanthosoma sp. Reko }\end{array}$ \\
\hline Güinar & \multicolumn{2}{|c|}{ Sida rhombifolia L } \\
\hline Corongoro & \multicolumn{2}{|c|}{ Zizyphus sonorensis Wats } \\
\hline Chucumpún & \multicolumn{2}{|c|}{ Cyrtocarpa procera H.B.K. } \\
\hline Tarape & \multicolumn{2}{|c|}{ Lantana involucrata $\mathrm{L}$} \\
\hline $\begin{array}{l}\text { Huizache o } \\
\text { Huisache }\end{array}$ & $\begin{array}{l}\text { Acacia farnesiana L.Willd. } \\
\text { Caesalpina cacalaco } \\
\text { Humb. }\end{array}$ & Golmania foetida Jacq Stand \\
\hline Huisache bola & \multicolumn{2}{|c|}{ Caesalpinia cacalaco Humb. } \\
\hline Huisache costeño & \multicolumn{2}{|c|}{ Acacia Hindsii Benth.Standley } \\
\hline Huisache conchilla & \multicolumn{2}{|c|}{ Goldmania foetida Jacq } \\
\hline Huisache tepame & \multicolumn{2}{|c|}{ Acacia cymbispina Sprague } \\
\hline Shure & \multicolumn{2}{|c|}{ No citada anteriormente } \\
\hline Golondrina & $\begin{array}{l}\text { Boerhava erecta L. } \\
\text { Matuda } \\
\text { Euphorbia hirta L. } \\
\text { Standley } \\
\text { Euphorbia thymifolia } \\
\text { Burm. } \\
\text { Pilea muscosa Lindl. } \\
\text { Ramírez y Alcocer }\end{array}$ & $\begin{array}{l}\text { Euphorbia glomerifera Millps. } \\
\text { Euphorbia adenoptera Benth. Pilea } \\
\text { microphylla Liebm. Ramírez. }\end{array}$ \\
\hline $\begin{array}{l}\text { Golondrina grande } \\
\text { Golondrina silvestre } \\
\text { Golondrina serrana } \\
\text { Golondrina de la sierra }\end{array}$ & \multicolumn{2}{|c|}{$\begin{array}{l}\text { Euphorbia hirta L González } \\
\text { Spermacoce tenuior L. } \\
\text { Euphorbia adenoptera Benht } \\
\text { Desmodium plicatum Schl }\end{array}$} \\
\hline $\begin{array}{l}\text { Rasca Viejo o } \\
\text { Rasca la Vieja }\end{array}$ & Cordia boissieri DC. & Curatella americana L. \\
\hline
\end{tabular}

\section{Discusión}

Las plantas medicinales siguen siendo en la actualidad una alternativa valiosa para el tratamiento de diversas enfermedades en muchos países del mundo (MORÓN et al., 2004). El Instituto Brasileño de Geografía y Estadística indica que el $40 \%$ de la población de ese país no tiene acceso a los medicamentos, 
razón por la cual las plantas medicinales son muy empleadas por sus pobladores (ALMEIDA et al., 2005). Algo similar ocurre en México, aunque no se dispone de las cifras exactas al respecto.

Se anota como importante la búsqueda de nuevos agentes antimicrobianos que ayuden a controlar el creciente incremento de cepas bacterianas patógenas de elevada resistencia a los antibióticos (VELAZQUES-MESA, 2005), la comunidad científica mundial evalúa cada día diferentes extractos, en su mayoría de origen vegetal, con la esperanza de que su acción antimicrobiana sea más potente, su espectro de actividad más amplio, produzcan menos efectos adversos y a la vez no induzcan el surgimiento de cepas bacterianas resistentes. La detección de nuevas fuentes terapéuticas parte en muchos casos del conocimiento y uso popular. La selección de las plantas es el primer paso antes de pasar a una etapa investigativa experimental en este campo.

El uso de encuestas es bastante frecuente en este tipo de trabajo, ya que suelen brindar informaciones útiles sobre el uso popular de las plantas con fines terapéuticos (SCULL et al., 1998; FUENTES et al., 2001). Cuando se carece de datos científicos que puedan brindar información en este sentido, es necesario obtenerlos a partir de fuentes primarias, es decir, encuestando directamente a personas conocedoras de la medicina tradicional y extrayendo de ahí la información necesaria para iniciar la colecta y estudio de las plantas (GUARRERA, 2005).

La encuesta empleada, de tipo dirigida o programada (SANDOVAL, 1998), tiene la característica de que en ella se entrevistan solo las personas conocedoras del problema. Esta metodología es muy empleada para detectar el conocimiento que tienen los pobladores de una localidad sobre las plantas medicinales (LA VOZ DE GALICIA.ES, 2009).

De las planillas de la encuesta (150 en total), solo se seleccionaron aquellas en las que se mencionaban plantas que por la descripción de su uso terapéutico se podía inferir que poseían algún tipo de propiedades antimicrobianas. Esa condición redujo el número de planillas a 30 , a pesar de lo cual las posibilidades de un efecto real de curación o mejoría con el uso de esas 
plantas sigue siendo una valoración empírica. La ausencia de un diagnóstico clínico previo o de laboratorio de las enfermedades presentes en las personas que las emplean, ofrece un rango de agentes etiológicos muy variado como bacterias, hongos y parásitos (GUERRA et al, 2004; HERNÁNDEZ y RODRíGUEZ, 2001) incluso otras enfermedades de causa no microbiana como problemas alérgicos, intoxicaciones o enfermedades de origen metabólico.

El hecho de que ninguna de estas plantas proceda de establecimientos comerciales para tales fines (comercio de yerberos) se debe a que las encuestas se realizaron en zonas rurales y las personas involucradas conocen las plantas por sus características morfológicas y prefieren recogerlas directamente del campo.

Cinco de las nueve plantas recomendadas se emplean para el tratamiento de diarreas, lo que es lógico, ya que las enfermedades diarreicas agudas (EDA) se encuentran entre las enfermedades infecciosas más frecuentes que afectan a los países en vías de desarrollo. La existencia de malas condiciones higiénico sanitarias entre la población de escasos recursos, favorece los brotes de este tipo de enfermedades (MECKES, et al., 1997). Esto hace que las plantas empleadas para el tratamiento de esta patología tengan una gran solicitud y amplia difusión (JIMÉNEZ et al., 2000). El uso de las plantas con fines antidiarreicos es uno de los más comunes para este recurso natural (SCULL et al., 1998).

Todas las plantas recomendadas para el tratamiento de la diarrea se emplean en forma de infusión. Ellas son: K. humboldtiana, W. indica, L. alba, A. farnesiana y $D$. minutifolia. Zizyphus amole se usa para tratar heridas (MARTíNEZ, 1996), este autor confirma los resultados obtenidos en la presente investigación, en la cual se encontró que los pobladores de Tierra Caliente emplean la corteza de $Z$. amole reducida a polvo para curar heridas e infecciones de la piel. También emplean la corteza de $D$. minutifolia para curar la diarrea. Algunas de estas plantas se utilizan como combustible para leña o para la preparación de carbón y según GONZÁLEZ et al. (2006) entre las plantas forrajeras que más se emplean como leña en el Estado de Michoacán 
están $D$. minutifolia y $Z$. amole. La tala no controlada y el uso no sostenible de estas plantas pueden poner en peligro su uso en medicina tradicional y su conservación (GONZÁLEZ y GUTIÉRREZ, 2005).

La amplitud territorial y variedad climática de la nación mexicana hacen que en exista una gran diversidad de plantas que se emplean en la medicina tradicional de este país, que en ocasiones se usan con fines distintos en las diferentes Regiones y Estados y además se conocen por nombres diferentes. Cuando se analizaron los nombres comunes que reciben las nueve plantas seleccionadas de la encuesta para el presente trabajo y se buscó la sinonimia para las diferentes Regiones y Estados de México, fue sorprendente la diversidad de especies botánicas a las cuales se les dan los mismos nombres comunes.

Para conocer la sinonimia empleada para estas plantas en las distintas regiones mexicanas se consultaron las publicaciones de MARTíNEZ en 1994 y 1996, trabajos conocidos entre los más completos en el campo de la medicina natural mexicana. A partir de este autor, se pudo verificar la importancia de la identificación taxonómica de las plantas por la coincidencia o diversidad de nombres empleados en distintas regiones y Estados, como se relaciona en la Tabla 4.

Con el nombre común de Guayabillo se designan 10 especies distintas a Karwinskia humboldtiana en distintos estados de México. El Nombre común de Güinar se aplica a la especie Sida rhombifolia en Michoacán y al Corongoro en esa misma Región le corresponde otra especie del género Zizyphus ( $Z$. sonorensis). Hubo coincidencia en la especie denominada como Chucumpún $y$ en una de las tres especies conocida con el nombre de Huizache, aunque hay otros cuatro tipos de Huizache con otras especificaciones. Existen al menos ocho especies diferentes que se conocen como Golondrina y existen cuatro tipos de variantes: Golondrina grande, Golondrina serrana, Golondrina de la sierra y Golondrina silvestre, para los que se pudieron encontrar identificaciones de cinco especies del género Euphorbia, dos especies 
pertenecientes a Pilea y otras dos especies de los géneros Boerhavia y Desmodium.

En el caso del Shure (Diphysa minutifolia) esta especie no aparece indicada como medicinal en el estudio de MARTíNEZ (1996). Esta misma planta en el estado de Michoacán, se conoce con el nombre de Churi (GONZÁLEZ et al., 2006).

En la planta conocida como Rasca Viejo o Rasca la Vieja, además de identificar a Curatella americana, aparece la especie Cordia boissieri DC. Al realizar la clasificación taxonómica de las nueve plantas en estudio, tres de ellas identificadas como K. humboldtiana, C. procera, Z. amole y D. minutifolia no aparecen reportadas anteriormente como plantas medicinales 0 con propiedades antibacterianas en el Estado de Guerrero, aunque existen otras especies de estos géneros que se emplean con fines terapéuticos.

\section{Referencias}

ALMEIDA, A.P.; CHÁVEZ, D.S.A.; RODRÍGUEZ, V.F.; PASSINI, A.M.; MORALES, S.R. 2005. Pharmaceutical care in phytotherapy: a tool to promote the education and to increase the interest on phytomedicine: Rev Cubana Plant Med. [online] sep-dic. 5 (3), hhtp:// bus.sld.cu/revistas/pla/vol 10_esp_05/pla_01405.hit \# Pla 1 [Consultado 23 Enero 2007]

CÁCERES, A. 2005. Desarrollo de tecnología de cultivo de plantas medicinales nativas y producción de fitoterápicos en Centro América. Rev. Cubana Plant Med. Suplemento Especial [online] sep-dic. 5 (3), hhtp:// bus.sld.cu/revista/pla/vol 10_esp_05/pla_01405. hit \# Pla 1 [Consultado 23 Enero 2007]

ECHAVARÍA, M.; LÓPEZ, R.; FERRER, Y.; CASADO, C.; MIRANDA, M. 2006. El Centro Nacional de Información de Plantas Medicinales y Productos Naturales como promotor del uso racional de la medicina natural. Memorias de la $1^{\text {a }}$ Jornada Científica de Fitoterapia CENSAM, 9 -10 nov, La Habana, Cuba.

FUENTES, V.R. ; GRANDA, M. ; LEMES, H.C. 2001. Estudio fenológicos en plantas medicinales XII. Rev Cubana Plan Med. 6 (3):87 -92. 
GONZÁLEZ, J.C.; AYALA, A.; GUTIÉRREZ, E. 2006ª . Determinación de fenoles totales y taninos condensados en especies arbóreas con potencial forrajero de la región Tierra Caliente Michoacán, México. Livestock Res Rural Development 18 (11):1-9.

GONZÁLEZ, J.C.; GUTIÉRREZ, V.E. 2005. Usos tradicionales de algunos árboles forrajeros de la región de Tierra Caliente Michoacana. Memorias del II Encuentro Nacional de Ecotecnias, Morelia, Michoacán, 7 al 10 de junio del 2005, México.

GUARRERA, P.M. 2005. Traditional phytotherapy in central Italy (Marche, Abruzzo and Latium). Fitoterapia 76:1-25.

GUERRA, M.; VEJA, R.; RIVERO, R.; MENÉNDEZ, R.; GUTIÉRREZ, A.; GUERRA, I. 2004. Actividad Antimicrobiana y toxicidad de un extracto acuoso de Boerhavia erecta L. Rev Cubana Plant Med. 9 (1):1-8.

HERAS-MEDINA, A. 1997. Conocimiento sobre plantas medicinales en una comunidad estudiantil de Atlautla, estado de México, México Tlahui-Medic. 3 (I):19-24.

HERNÁNDEZ, L.; RODRíGUEZ, M. 2001. Actividad antimicrobiana de plantas que crecen en Cuba. Rev Cubana Plant Med. 2:44- 47.

INEGI - Instituto de Estadística Geografía e Informática. 2000. Indicadores de Población y Vivienda XII Censo General de Población y Vivienda de Estados Unidos Mexicanos: P 1-200

JIMÉNEZ, M.; RUIZ, R.; NAVARRO, A.; HERNÁNDEZ, J.M.; REYES, R. 2000. Plantas usadas en México para curar la diarrea (Estudio Químico y Bacteriológico). Rev Cubana Quím. 13 (2):4 - 22.

LA VOZ DE GALICIA.ES. Encuestas familiares y un archivo sobre plantas medicinales. [serial online] 2009 [citado 12 nov 2009]; 6 (5) 2009. Disponible en http://www.lavozdegalicia.com/ferrol/2009/05/06/0003 7698262.htm

MARTíNEZ, M. 1994. Catálogo de nombres vulgares y científicos de plantas mexicanas. Editorial Fondo de Cultura Económica, quinta edición. México.

MARTíNEZ, M. 1996. Las Plantas Medicinales de México. $7^{a}$ Reimpresión. Edit. Botas S.A. México.

MECKES, M.; DAVID, A.D.; NAVA, V.; JIMÉNEZ, A. 2004. Activity of some Mexican medicinal plant extracts on carrageenan-induced rat paw oedema. Phytomed 11:446-451. 
MORÓN, F.; SIERRA, P.; VILLÁN, J.; MARTÍNEZ, M.J. 2004. Programa de Medicina Tradicional herbolaria en Cuba. Las plantas medicinales en la terapéutica Rev Cubana Med Gen Integral 7 (3):276-204.

MORÓN, F. 2006. Plantas medicinales. Datos metodológicos: siete respuestas sobre experiencia cubana. Salud y Vida (Infomed) http//bus sld. Revists/pla/vol 10-esp-05/pla-01405.hit \# pla 1 [consultado miércoles 20 Dic 2006]

OPS, Washington EUA: 225p.PINEDA, E.B., DE ALVARADO, E.L. y DE CANALES, H. (2000). Metodología de la investigación, Manual para el desarrollo de personal de salud. $2^{\text {da }}$ Edición.

SALGADO, R.; CALDERÓN, M.J.C.; SERRATO, E.E.; BELLO, G.M.A.; LÓPEZ, E.C.; COVIÁN, N.F.; LOEZA, L.P.D.; MORALES, L.M.E.; DAMIÁN, B.L.M.; MARTíNEZ, M.M. 2005. Actividad biocida de extractos vegetales de especies etnobotánicas de la región centro-occidente de México. Rev Cubana Plant Med, Número Especial. [online] sep-dic. 5 (3), hhtp:// bus.sld.cu/revista/pla/vol 10_esp_05/pla_01405.hit \# Pla 1[consultado 23 Enero 2007]

SCULL, L.R.; MIRANDA, M.M.; INFANTE,L.R. 1998. Plantas Medicinales de Uso Tradicional en Pinar del Río. Estudio Etnobotánico. Rev Cubana Farm 32 (1):57- 62.

VELÁZQUES-MESA, M.E. 2005. Surgimiento y diseminación de Staphylococcus aureus meticilin-resistente. Salud Pública Méx. 47:381- 387. 